MKG-Chirurg 2009 - 2:69-70

DOI 10.1007/s12285-009-0084-3

Online publiziert: 15. Mai 2009

(c) Springer Medizin Verlag 2009

\author{
E. Esser ${ }^{1} \cdot$ H.-P. Ulrich ${ }^{2}$ \\ ${ }^{1}$ ICOS ImplantatCentrum Osnabrück \\ 2 MKG-Chirurgie LindenArcaden, Lübeck
}

\title{
Leitlinien und evidenzbasierte Medizin
}

kommission der DGMKG installiert. Aktuell sind zwei Leitlinien der höchsten Stufe (S3), acht der Stufe $S_{2}$ und acht der Stufe $S_{1}$ auf der AWMF-Leitlinienseite (http://www.awmf-leitlinie.de) eingestellt. Weiterhin sind vier Leitlinien mit S1-Niveau auf den Seiten der DKG (http://www.krebsgesellschaft.de/wub_ llkurz_2008,78263.html\#b) zu sehen.

Ein großes Problem der Leitlinienarbeit besteht darin, dass diese einerseits sehr aufwendig ist, andererseits Ergebnisse erst spät oder gar nicht gesehen werden. Dadurch, dass Leitlinien mit benachbarten Fächern abzustimmen sind, bedarf es hierzu großen diplomatischen Geschickes. Man könnte überspitzt formulieren: In Bereichen, in denen nicht gearbeitet wird, kann viel geschrieben werden. Oder anders formuliert: In diesem Bereich sind chirurgische Fächer nichtchirurgischen unterlegen.

\section{(?) Alle Mitglieder der DGMKG werden gebeten, die Leitlinien- kommission zu unterstützen}

Umso mehr ist es unsere Aufgabe, die Sensibilität aller Mitglieder der DGMKG zu wecken, um den Leitlinienkoordinator bei seiner Arbeit zu unterstützen. Ist einmal etwas festgeschrieben, kann dies nur schwer und in mühevoller Überzeugungsarbeit geändert werden.

Aus eigener Mitarbeit an der Leitlinie Wurzenspitzenresektion wissen die Unterzeichner, wie schwierig die Tätigkeit für den Leitlinienkoordinator (Herrn Prof. Dr. Dr. Martin Kunkel) war und wie mühsam das Ringen selbst um die Inter- punktion gelegentlich wurde. Auf einen entscheidenden Halbsatz in dieser Leitlinie wurde vom ehemaligen Präsidenten anlässlich unserer Praxisführungsseminare mehrfach verwiesen.

In der aktuellen Ausgabe wird mit der Leitlinie zur Weisheitszahnentfernung ein typisches Beispiel präsentiert.

Die WHO definiert Leitlinien wie folgt: „... systematisch entwickelte Feststellungen mit dem Ziel, die Entscheidung von Ärzten und Patienten über eine angemessene Gesundheitsversorgung für spezifische klinische Situationen zu unterstützen. Sie definieren damit eine Korrektur von Diagnosen und Behandlungspfaden, von dem im Einzelfall abgewichen werden kann und manchmal sogar abgewichen werden muss. " Die Berücksichtigung dieser Leitliniendefinition ist auch unter juristischen Gesichtspunkten für uns dringlich; dieses Feld muss kompetent bestellt werden.

Dass wir unsere bisherigen Ziele in der jetzigen Form erreichen konnten, ist der unermüdlichen Arbeit von Herrn Prof. Dr. Dr. Hans Pistner geschuldet, und ihm gilt an dieser Stelle der Dank sowohl der Herausgeber als auch aller Mitglieder der DGMKG.

Diese Arbeit ist jedoch nur möglich durch den Einsatz der Leitlinienkoordinatoren, denen in gleicher Form hier zu danken ist, sowie auch der anderen Kollegen, die mitarbeiten.

Wir möchten Sie dringend auffordern, die Leitlinienkommission zu unterstützen, und wären dankbar, wenn Sie bei persönlichen Ansprachen kurzfristig zur Verfügung stehen könnten. 
Wenn wir nicht aufpassen, passen andere auf, und Teile unseres Faches werden eingeschränkt und/oder gehen verloren.

Wir danken bereits jetzt für Ihr Verständnis und Ihre Kooperation und verbleiben

mit besten kollegialen Grüßen

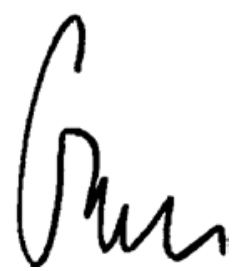

Elmar Esser

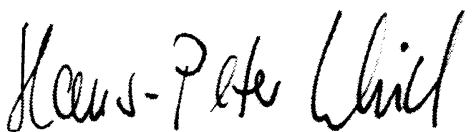

Hans-Peter Ulrich

\section{Korrespondenzadressen}

\section{Prof. Dr. Dr. E. Esser}

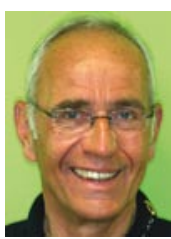

ICOS ImplantatCentrum

Am Finkenhügel 3,

49076 Osnabrück

e.esser@icosnet.de

\section{Dr. Dr. H.-P. Ulrich}

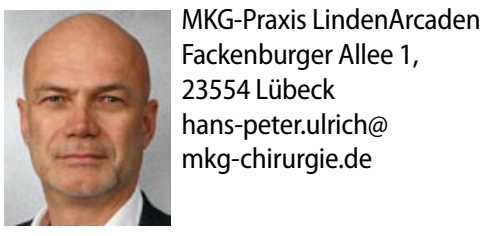

"Der MKG-Chirurg" bietet Ihnen umfassende und aktuelle Beiträge zu interessanten Themenschwerpunkten aus allen Bereichen der Mund-, Kiefer- und Gesichtschirurgie.

Wir haben die Jahrgänge 2008/2009 im Überblick für Sie zusammengestellt:

\section{MerkG-Chirurg \\ 2008 \\ - 01/08 3-D-gestützte Verfahren in der Implantologie \\ - 02/08 Schlafbezogene Atmungsstörungen \\ - 03/08 Ästhetische Chirurgie}
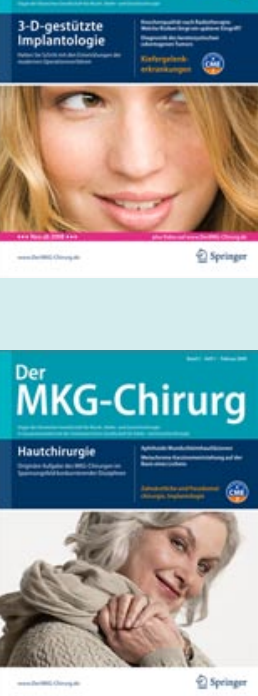
Strotive

2009

- 01/09 Hautchirurgie

02/09 Leitlinien und evidenzbasierte Medizin

- 03/09 Onkologie

- 04/09 Botulinumtoxin und Filler
(Änderungen vorbehalten)

Bestellen Sie einzelne Ausgaben oder abonnieren Sie die Zeitschrift zum Preis von 149 EUR pro Jahr (zzgl. Versandkosten, Ermäßigung für Ärzte in Fort- und Weiterbildung).

Unser Kundenservice steht Ihnen für Fragen und Informationen gerne zur Verfügung:

Springer Customer Service Center Haberstrasse 7, 69126 Heidelberg

Tel: +49(6221)345-4303, Fax: +49(6221)345-4229

E-Mail: Leserservice@springer.com

Eine interessante Lektüre wünscht Ihnen

Ihre Redaktion

Fachzeitschriften Medizin/Psychologie 